\title{
Bounds and prices of currency cross-rate options
}

\author{
San-Lin Chung, Yaw-Huei Wang * \\ Department of Finance, National Taiwan University, No. 1, Section 4, Roosevelt Road, Taipei 10617, Taiwan, ROC
}

Received 27 February 2006; accepted 30 January 2007

Available online 14 September 2007

\begin{abstract}
This paper derives the pricing bounds of a currency cross-rate option using the option prices of two related dollar rates via a copula theory and presents the analytical properties of the bounds under the Gaussian framework. Our option pricing bounds are useful, because (1) they are general in the sense that they do not rely on the distribution assumptions of the state variables or on the selection of the copula function; (2) they are portfolios of the dollar-rate options and hence are potential hedging instruments for cross-rate options; and (3) they can be applied to generate bounds on deltas. The empirical tests suggest that there are persistent and stable relationships between the market prices and the estimated bounds of the cross-rate options and that our option pricing bounds (obtained from the market prices of options on two dollar rates) and the historical correlation of two dollar rates are highly informative for explaining the prices of the cross-rate options. Moreover, the empirical results are consistent with the predictions of the analytical properties under the Gaussian framework and are robust in various aspects.
\end{abstract}

(c) 2007 Elsevier B.V. All rights reserved.

JEL classification: F3; F4; G1

Keywords: Option pricing; Option bounds; Exchange rates; Cross-rate; Correlation; Copulas

\section{Introduction}

In the option pricing literature, researchers are not only interested in pricing, but also in bounding the option values. There are many useful techniques that can be employed to derive option pricing bounds. For example, Merton (1973), Garman (1976), Levy (1985), and Grundy (1991) use the arbitrage-free approach to derive option pricing bounds. Ritchken (1985), Ritchken and Kuo (1989), Basso and Pianco (1997), Mathur and Ritchken (2000), and Ryan (2003) use linear programming methods to derive option pricing bounds. In addition to the above two types of techniques, some other approaches, such as optimization methods and restrictions on the volatility of the pricing kernel, have also been used in the literature.

\footnotetext{
* Corresponding author. Tel.: +886283695581.

E-mail address: yhwang@management.ntu.edu.tw (Y.-H. Wang).
}

Most, if not all, of the previous studies derive option pricing bounds by directly using the price information (such as the price distribution or price process) of the underlying asset. In contrast to the previous literature, this study uses the option prices of the related dollar rates to derive the pricing bounds for the cross-rate option. In other words, we bound cross-rate option values using the market prices of the dollar-rate options. ${ }^{1}$ In this sense, the idea of this paper is close to that in the static hedge literature (see Carr et al., 1998), whereby the exotic options are priced (and hedged) in terms of the prices of standard options.

Since there is a triangular relationship between the foreign exchange rates among three currencies, Taylor and

\footnotetext{
${ }^{1}$ The motivation for doing this is as follows. It is generally observed that options on dollar-denominated exchange rates are traded under satisfactory liquidity, while cross-rate option markets are much less liquid. Thus, the pricing bounds obtained from the liquid market prices of dollar-rate options are useful for pricing, hedging, and arbitraging.
} 
Wang (2005) show that it is plausible to estimate risk-neutral densities (RNDs) and option prices of a cross-rate under the US dollar measure ${ }^{2}$ using the market prices of two related dollar-rate options. Instead of directly exploring the option pricing formula, this study (also using the dollar risk-neutral measure) derives the pricing bounds for cross-rate options by utilizing the exchange option pricing bounds implied in the copula theory. ${ }^{3}$ Some analytical properties of the bounds under the Gaussian framework are also presented in this paper. Compared with the previous studies, our objective is not to derive tight bounds, but rather to generate informative bounds from useful market price information (dollar-rate option prices).

Our option pricing bounds contribute to the literature in at least three aspects. First of all, although our pricing bounds are not tight, they are general in the sense that they do not rely on the distribution assumptions of the state variables or on the selection of a copula function. Secondly, our pricing bounds have economic meanings, because they are portfolios composed of the dollar-rate options (and sometimes also composed of spot dollar rates) and hence provide potential hedging instruments for crossrate options. Finally, our pricing bounds are also useful for generating bounds on deltas.

The empirical tests of our pricing bounds are conducted using the prices of options on foreign exchange rates among the US dollar, euro, and pound sterling. We first show that there are strong and stable relationships between the market prices of cross-rate options and the pricing bounds obtained from the market prices of options on the two dollar rates. Both of the above finding and the analytical analysis in Section 2 motivate us to run the regression models to measure the extent where the cross-rate option prices can be explained by our pricing bounds and the correlation between the two dollar rates.

Our empirical results indicate that the pricing bounds estimated from option prices of two dollar rates and the correlation of two dollar rates can provide highly significant information (about $85 \%$ ) for explaining the cross-rate option prices across deltas. Our results are immune to the assumption of the RND distribution for the dollar rates, the market volatility level, and the change in the curvature of the implied volatility function. Finally, we demonstrate how to calculate bounds on deltas using our pricing bounds.

\footnotetext{
${ }^{2}$ Both Taylor and Wang (2005) and this paper commence the analyses under the foreign (dollar) risk-neutral measure to price the cross-rate options. As suggested by Bakshi et al. (forthcoming), it may be better to specify the generic pricing kernels in each country to derive the prices of derivatives. Nonetheless, Taylor and Wang (2005) analytically show that the prices of derivatives under different measures are equivalent when the law of one price holds. In order to utilize the concept of the exchange option, this article formulates the pricing problem under the dollar measure.

${ }^{3}$ The details of the copula theory can be found in Joe (1997) and Nelsen (1999). Cherubini et al. (2004) first apply the copula theory to derive the pricing bounds for the exchange options.
}

The remainder of this paper is organized as follows. Section 2 derives option pricing bounds for the cross-rate option, presents their analytical properties under the Gaussian framework, and shows how to bound the delta of the cross-rate option using the derived pricing bounds. Data and the empirical methodologies for generating the risk-neutral densities and option pricing bounds are presented in Section 3. Section 4 discusses the empirical results, while Section 5 concludes the paper.

\section{Bounds of the price and delta of the cross-rate option}

\subsection{Bounds of the price of the cross-rate option}

By applying the Fréchet bounds in the copula theory, Cherubini et al. (2004) show that the super-replication bounds of the option to exchange one asset for the other asset are composed of the prices of the univariate options on the two individual exchanged assets. ${ }^{4}$ We first show that the payoff of a cross-rate option under the dollar measure is equivalent to that of an exchange option where the two underlying risky assets are the corresponding dollar rates. Following the same logic as in Cherubini et al. (2004), we use the risk-neutral pricing approach to derive the pricing bounds for the cross-rate option.

Consider options whose payoffs depend on the exchange rates among the following three currencies: US dollars $(\$$, USD), British pounds ( $£$, GBP), and euros ( $€$, EUR). We denote the dollar price of one pound at time $t$ by $S_{t}^{\$ / E}$ and likewise the dollar price of one euro at the same time is denoted by $S_{t}^{\$ / \epsilon}$. The cross-rate price of one pound in euros is then given by $S_{t}^{\ell / £}=S_{t}^{\$ / £} / S_{t}^{\$ / \epsilon}$ under the no-arbitrage argument.

Now consider a European call option where the holder has the right to buy $£ 1$ for $€ K$ at time $T$. Under the dollar measure (or from the viewpoint of US residents), the above option is identical to an option to exchange $K S_{T}^{\$ / \epsilon}$ dollars for $S_{T}^{\$ / £}$ dollars at time $T$. Hence, a cross-rate call option under the dollar measure is equivalent to an option to exchange one asset for the other asset and its dollar payoff equals $\max \left(S_{T}^{\$ / \mathfrak{t}}-K S_{T}^{\$ / \epsilon}, 0\right)$. This payoff can be rearranged as follows:

$S_{T}^{\$ / £}-\max \left[\min \left(S_{T}^{\$ / £}, K S_{T}^{\$ / \epsilon}\right), 0\right]$.

Hence, the current dollar price of an exchange option is determined by the risk-neutral pricing approach as follows:

$\mathrm{Call}_{\$}^{€ / £}=S_{t}^{\$ / £} \mathrm{e}^{-r_{\mathfrak{£}}(T-t)}-\mathrm{Call}_{\text {min }}\left(S^{\$ / £}, K S^{\$ / €}, 0, t, T\right)$,

where Call $\mathrm{min}_{\min }\left(S_{1}, S_{2}, 0, t, T\right)$ represents the price at time $t$ of a call option on the minimum of $S_{1}$ and $S_{2}$ with strike price 0 and maturity time $T$. Applying the Fréchet bounds in the

\footnotetext{
${ }^{4}$ However, Cherubini et al. (2004) only derive the lower bound for one particular probability condition. There should be an alternative formula applied to the other probability condition. See our Eq. (4) for these two probability conditions.
} 
copula theory, we are able to derive the upper (lower) bound of the minimum call option price and thus the lower (upper) bound of the cross-rate option price as follows.

Proposition 1. The upper bound of the cross-rate option price in dollars is

$\mathrm{Call}_{\S}^{€ / \mathfrak{£}^{+}}=\operatorname{Call}\left(S^{\$ / £}, K^{* *}, t, T\right)+K \operatorname{Put}\left(S^{\$ / €}, K^{\prime \prime}, t, T\right)$,

where $K^{* *}$ is a constant satisfying that $\bar{F}_{S^{s / \mathrm{t}}}\left(K^{* *}\right)+$ $\bar{F}_{K S^{s / e}}\left(K^{* *}\right)=1, \bar{F}_{i}(x)=1-F_{i}(x), F_{i}(x)$ is the cumulative distribution function, and $K^{\prime \prime}=K^{* *} / K$. Let $K^{*}$ be a constant which solves $\bar{F}_{S^{s / \mathrm{t}}}\left(K^{*}\right)=\bar{F}_{K S^{s / \epsilon}}\left(K^{*}\right)$. The lower bound of the cross-rate option price in dollars is thus

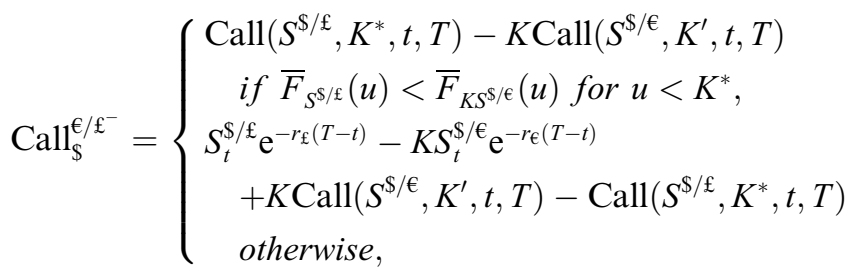

where $K^{\prime}=K^{*} / K$.

Proof. Please see Appendix A.

From Eqs. (3) and (4), we observe that our pricing bounds for cross-rate options are portfolios of the corresponding dollar-rate options (and may also be of the spot assets). Therefore, different from most option pricing bounds in the literature, the derived pricing bounds have economic meanings. Moreover, the derivation of our cross-rate option pricing bounds does not rely on the distribution assumption of two dollar rates and the selection of an appropriate copula function. Therefore, one can apply the technique utilized here to derive the price bounds for any European-style derivatives whose payoffs can be rearranged as the same type as that of an exchange option.

Since the cross-rate is completely determined by the other two dollar rates under the triangular arbitrage relation, a natural question to ask is how the payoff of the cross-rate option is related to the payoffs of the other two dollar-rate options. If this relationship can be specified, one is able to apply the spanning approach of Bakshi and Madan (2000) to price (or to provide pricing bounds for) the cross-rate options using the prices of two dollarrate options. In Corollary 1 we show that the correlation options considered in Bakshi and Madan (2000) provide a lower bound for the cross-rate option price. The proof of Corollary 1 is available from the authors upon request.

Corollary 1. The price of a cross-rate call option with strike price $K$ is bounded below by the price of a correlation option with the following payoff:

$\max \left(S_{T}^{\$ / £}-K_{x}, 0\right) \times \max \left(1 / S_{T}^{\$ / \epsilon}-1 / K_{y}, 0\right)$,

where $K=K_{x} / K_{y}$.
Note that Corollary 1 relates the lower pricing bound of a cross-rate call option to the price of a dollar-rate call (with a payoff of $\max \left(S_{T}^{\$ / \mathrm{t}}-K_{x}, 0\right)$ ) and the price of a dollar-rate put (with a payoff of $\max \left(K_{y}-S_{T}^{\$ / \epsilon}, 0\right)$ ). Therefore, Corollary 1 suggests that the dollar-rate option prices (and hence our pricing bounds) may be informative for explaining the cross-rate option prices. Later in Section 3 we will run a regression model to investigate the explanatory power of our pricing bounds.

\subsection{Analytical properties of the pricing bounds under the Gaussian framework}

When the two dollar rates follow a bivariate lognormal distribution, then under the triangular arbitrage relation the cross-rate also follows a lognormal distribution. Thus, there exist closed-form solutions for the option prices of two dollar rates and our pricing bounds. To have some insights on our pricing bounds, we investigate the analytical properties of these bounds when the two dollar rates follow a bivariate lognormal distribution.

Denote the closed-form solutions of two dollar-rate option prices at time $t$, denominated in US dollars, as $C_{\mathrm{BS}}\left(S_{t}^{\S / £}, K, r_{\S}, r_{£}, \sigma_{\$ / £}, \tau\right) \quad$ and $\quad C_{\mathrm{BS}}\left(S_{t}^{\$ / \epsilon}, K, r_{\$}, r_{€}, \sigma_{\$ / \epsilon}, \tau\right)$, respectively. Proposition 2 shows that the upper and lower bounds have closed-form solutions under the bivariate lognormal distribution assumption.

Proposition 2. Assume that two dollar rates $S^{\$ / \epsilon}$ and $S^{\$ / \epsilon}$ follow a bivariate lognormal distribution with a correlation coefficient of $\rho$ and volatilities per year of $\sigma_{\$ / £}$ and $\sigma_{\$ / €}$, respectively. The upper and lower bounds, denominated in euros, of the cross-rate call option with a strike price of $K$ thus have closed-form solutions of $C_{\mathrm{BS}}\left(S_{t}^{\in / \mathfrak{t}}, K, r_{€}, r_{\mathfrak{£}}, \sigma_{\$ / \mathfrak{f}}+\right.$ $\left.\sigma_{\$ / €}, \tau\right)$ and $C_{\mathrm{BS}}\left(S_{t}^{\epsilon / £}, K, r_{€}, r_{\mathfrak{£}},\left|\sigma_{\$ / \mathfrak{f}}-\sigma_{\$ / €}\right|, \tau\right)$, respectively.

Proof. Please see Appendix B.

Under the bivariate lognormal distribution assumption, the triangular arbitrage relation implies that the cross-rate option price, denominated in euros, is $C_{\mathrm{BS}}\left(S_{t}^{\mathrm{\epsilon} / \mathfrak{f}}, K, r_{€}, r_{\mathfrak{f}}\right.$, $\left.\sigma_{€ / \mathfrak{f}}, \tau\right)$, where $\sigma_{\epsilon / \mathfrak{\epsilon}}^{2}=\sigma_{\$ / \mathfrak{\epsilon}}^{2}+\sigma_{\$ / \epsilon}^{2}-2 \rho \sigma_{\$ / \mathfrak{t}} \sigma_{\$ / \epsilon}$. Therefore, Proposition 2 is intuitively true, because $\left|\sigma_{\$ / £}-\sigma_{\$ / \epsilon}\right| \leqslant$ $\sigma_{€ / £} \leqslant \sigma_{\$ / £}+\sigma_{\Phi / €}$. When the correlation between two dollar rates is higher (lower), the cross-rate option price is closer to the lower (upper) bound. Moreover, Proposition 2 implies that when the implied volatility curves of two dollar-rate option prices are flat, then the implied volatility curves of our upper and lower bounds for cross-rate options are also flat.

\subsection{Bounds on the delta of the cross-rate option}

Given the estimated pricing bounds in terms of implied volatilities, it is plausible to derive bounds on the cross-rate option's delta using Proposition 5 of Bergman et al. (1996). Assume that the volatility function, $\sigma(s, t)$, is a function of the underlying asset price $s$ and time $T$ only. Let $\underline{\sigma}$ and $\bar{\sigma}$, 
respectively, denote the lower and upper bounds on volatility, $c(s, t)$ and $c_{1}(s, t)$, respectively, represent the market (or accurate) call price and its delta, and $c^{\mathrm{bs}(\sigma)}$ and $c_{1}^{\mathrm{bs}(\sigma)}$, respectively, stand for the Black-Scholes call price and its delta. Bergman et al. (1996) derive bounds on the option's delta as follows.

Proposition 5 of Bergman et al. (1996). If for all $s$ and $t, \underline{\sigma}(t) \leqslant \sigma(s, t) \leqslant \bar{\sigma}(t)$, then $c_{1}^{\mathrm{bs}(\bar{\sigma})}\left(s^{\prime \prime}, t\right) \leqslant c_{1}(s, t) \leqslant c_{1}^{\mathrm{bs}(\bar{\sigma})}\left(s^{\prime}, t\right)$,

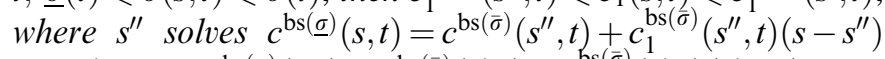
and $s^{\prime}$ solves $c^{\mathrm{bs}(\underline{\sigma})}(s, t)=c^{\mathrm{bs}(\bar{\sigma})}\left(s^{\prime}, t\right)-c_{1}^{\mathrm{bs}(\bar{\sigma})}\left(s^{\prime}, t\right)\left(s^{\prime}-s\right)$.

The delta bounds of Bergman et al. (1996) are true for general Markovian diffusion processes. When the crossrate option's value today is known, Bergman et al. (1996) show that the bounds on its delta can be strengthened as follows.

Proposition 6 of Bergman et al. (1996). If for all $s$ and $t, \sigma(s, t) \leqslant \bar{\sigma}(t)$, then for any $s$ and $t$ such that one knows $c(s, t), c_{1}^{\mathrm{bs}(\bar{\sigma})}\left(s^{\prime \prime}, t\right) \leqslant c_{1}(s, t) \leqslant c_{1}^{\mathrm{bs}(\bar{\sigma})}\left(s^{\prime}, t\right)$, where $s^{\prime \prime}$ solves $c(s, t)=c^{\mathrm{bs}(\bar{\sigma})}\left(s^{\prime \prime}, t\right)+c_{1}^{\mathrm{bs}(\bar{\sigma})}\left(s^{\prime \prime}, t\right)\left(s-s^{\prime \prime}\right) \quad$ and $\quad s^{\prime} \quad$ solves $c(s, t)=c^{\mathrm{bs}(\bar{\sigma})}\left(s^{\prime}, t\right)-c_{1}^{\mathrm{bs}(\bar{\sigma})}\left(s^{\prime}, t\right)\left(s^{\prime}-s\right)$.

Our pricing bounds are directly applicable to Proposition 5 of Bergman et al. (1996), and thus the bounds on the deltas can be obtained straightforward. For instance, the implied volatility of our lower (upper) bound provides an estimate of $\underline{\sigma}(t)(\bar{\sigma}(t))$ for applying Proposition 5 of Bergman et al. (1996). When the market price of the cross-rate option is known, our upper bound can be used in conjunction with Proposition 6 of Bergman et al. (1996) to obtain tighter bounds for deltas. Later, we will calculate bounds on the deltas when our pricing bounds are applied to Propositions 5 and 6 of Bergman et al. (1996).

\section{Data and empirical methodologies}

\subsection{Data}

The primary data used in this article are daily option prices that are quoted as Black-Scholes implied volatilities for three currency options $(\$ / £, \$ / €$, and $€ / £)$. We make use of a confidential file of OTC option price mid-quotes, supplied by the trading desk of an investment bank in London. ${ }^{5}$ Our currency option data cover the period from 15 March 1999 to 11 January 2001. The OTC quotes are for all three foreign exchange options, recorded at the end of the day in London. The data include option prices for seven exercise prices, based upon "deltas" equal to $0.1,0.25,0.37$, $0.5,0.63,0.75$, and 0.9 . The time to maturity of the options

\footnotetext{
${ }^{5}$ Some settlement prices are available for cross-rate options traded in the Chicago Mercantile Exchange, but they correspond to almost no trading volume. Consequently, we rely on over-the-counter (OTC) option prices, with which we have the same time-to-maturity option data every day. To the best of our knowledge, such prices are not available in the public domain.
}

is one month, with which options in the OTC market are most frequently traded. We also use the spot exchange rates of $\$ / £, \$ / €$, and $€ / £$ and the euro-currency interest rates (proxies of risk-free rates) of $\$, £$, and $€$ recorded by DataStream as the inputs for all relevant calculations.

The summary statistics of the quoted implied volatilities show that all implied volatility functions exhibit a smile shape with the level for the $\$ / €$ options being the highest, while the level for the $\$ / £$ options are the lowest. The low standard deviations of the quotes imply that the levels of implied volatilities for these three exchange rate options do not change much as time goes. The skewness is positive and the kurtosis is close to 3 , which does not depend on the moneyness of the options.

\subsection{Empirical methodologies for generating the bounds}

Because $K^{*}$ and $K^{* *}$ are determined by the risk-neutral densities of two dollar rates, we use the observed market prices of European call options on $\$ / £$ and $\$ / €$ and a parametric distribution specification to estimate their riskneutral densities. Once the risk-neutral densities are obtained, $K^{*}$ and $K^{* *}$ can be calculated with a numerical method (such as the Newton-Raphson method) to solve $\bar{F}_{S^{s / \epsilon}}\left(K^{*}\right)=\bar{F}_{K S^{s / \epsilon}}\left(k^{*}\right)$ and $\quad \bar{F}_{S^{s / \varepsilon}}\left(K^{* *}\right)+\bar{F}_{K S^{s / \epsilon}}\left(K^{* *}\right)=1$, respectively. We are then able to price dollar-rate options with all strikes and get pricing bounds of the cross-rate options using Eqs. (3) and (4).

In this paper we use the generalized beta density of the second kind (GB2) to estimate the RNDs of two dollar rates. ${ }^{6}$ The GB2 density has few parameters, but it preserves many desirable properties: general levels of skewness and kurtosis are allowed, the shapes of the tails are fat relative to the lognormal density, and there are analytic formulae for the density, its moments, and the prices of options. Furthermore, the parameter estimation of the GB2 density is easy and the estimated densities are never negative. The details about the estimation of the GB2 density can be found in Bookstaber and McDonald (1987).

\section{Empirical results}

The empirical studies in this article contain four parts. We first analyze the properties of our pricing bounds and their relationships with the market prices of the cross-rate options. Second, we investigate the explanatory powers of the pricing bounds and the correlation between two dollar

\footnotetext{
${ }^{6}$ Many types of univariate RNDs have been proposed, including lognormal mixtures (Ritchey, 1990; Melick and Thomas, 1997), generalized beta densities (Bookstaber and McDonald, 1987), multi-parameter discrete distributions (Jackwerth and Rubinstein, 1996), and densities derived from fitting spline functions to implied volatilities (Bliss and Panigirtzoglou, 2002). Providing that options are traded for a range of exercise prices that encompass most areas of the risk-neutral distribution, it is documented that several flexible density families provide similar empirical estimates. The bounds estimated with the lognormal mixtures RNDs are compared with for the robustness check.
} 
Table 1

Summary statistics of the implied volatilities from the market prices and the estimated bounds

\begin{tabular}{llllllr}
\hline Delta & 0.9 & 0.75 & 0.63 & 0.5 & 0.37 & 0.25 \\
\hline Panel 1: Upper bounds & & & & & & 0.1 \\
Mean & 0.2087 & 0.2061 & 0.2056 & 0.2058 & 0.2064 & 0.2077 \\
Standard deviation & 0.0325 & 0.0329 & 0.0330 & 0.0331 & 0.0332 & 0.0333 \\
Panel 2: Market implieds & & & & & 0.2118 \\
Mean & 0.1054 & 0.0996 & 0.0980 & 0.0972 & 0.0979 & 0.0995 \\
Standard deviation & 0.0169 & 0.0173 & 0.0176 & 0.0176 & 0.0180 & 0.0180 \\
Panel 3: Lower bounds & & & & & 0.1050 \\
Mean & 0.0405 & 0.0319 & 0.0302 & 0.0315 & 0.0352 & 0.0396 \\
Standard deviation & 0.0095 & 0.0117 & 0.0124 & 0.0122 & 0.0113 & 0.0108 \\
\hline
\end{tabular}

This table consists of the summary statistics of the implied volatilities from the market prices and estimated upper and lower bounds of the cross-rate $€ / £$ options across deltas. The option bounds are estimated by calibrating Eqs. (3) and (4) with the option prices of two dollar rates, \$/£ and \$/€.

rates for the market prices of the cross-rate options. Third, some robust analyses for the accuracy of our results are provided. Finally, given the estimated price bounds of the cross-rate options, we demonstrate how to bound their deltas using the approach proposed by Bergman et al. (1996).

\subsection{Empirical pricing bounds of the cross-rate options}

In order to have a standardized comparison, all the market prices and pricing bounds are converted into the BlackScholes implied volatilities. The pricing bounds of the onemonth cross-rate options with seven different strike prices (deltas) are estimated every day. All lower bounds are determined by the second alternative of Eq. (4), because the implied volatilities of $\$ / €$ are always larger than those of $\$ / £$ during our sample period. ${ }^{7}$ The descriptive statistics of the estimated pricing bounds and the market implied volatilities across deltas are shown in Table 1 . We also show the evolution of the estimated pricing bounds and the market implied volatilities in Fig. 1. As the patterns across deltas are very similar, Fig. 1 presents the result with a delta of 0.5 only.

As shown in Fig. 1, the market implied volatility always lies within the estimated bounds, and the evolution of the market implied volatility of the cross-rate $(€ / £)$ option exhibits a similar pattern to those of the estimated bounds. As the foreign exchange market became more volatile from 1999 to 2000 , the bound range, defined as the difference between the upper bound and the lower bound, turned wider as time went by during the period.

Table 1 suggests that the level, the mean, and the volatility of the upper bounds are almost the same across deltas with an extremely shallow smile. In contrast, the lower bound and the market implied volatilities exhibit clearer smile shapes across deltas with the lower bound smile being deeper than the market implied smile.

To explore the relationships between the option market prices and the estimated bounds, we further look at the

\footnotetext{
${ }^{7}$ The analytical properties of our lower bound under the Gaussian framework suggest that if the volatility of $\$ / €$ is greater than that of $\$ / £$, then $\bar{F}_{S^{s / \varepsilon}}(u)>\bar{F}_{K S^{s / \epsilon}}(u)$ for $u<K^{*}$ and vice versa.
}

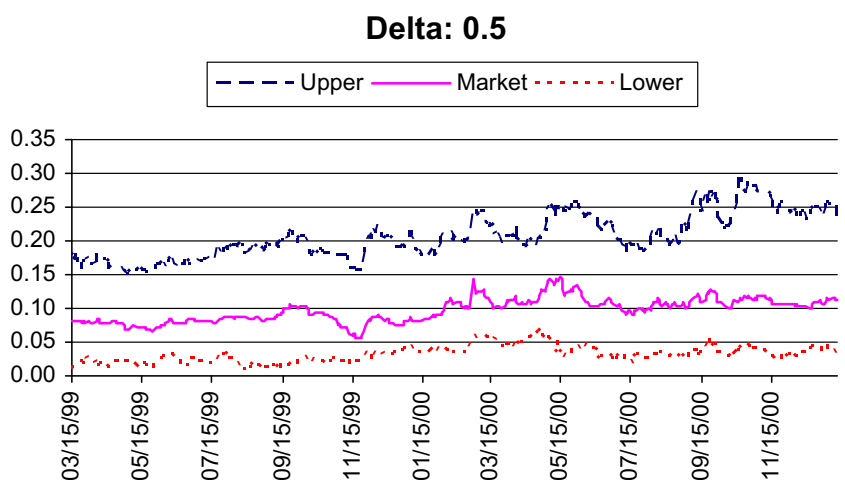

Fig. 1. The implied volatilities from market prices and the estimated bounds for cross-rate options. This figure shows the evolution of the Black-Scholes implied volatilities from the market prices and the estimated bounds of the cross-rate $(€ / £)$ options with the delta of 0.5 . The cross-rate option pricing bounds are estimated by calibrating Eqs. (3) and (4) using the option prices of two dollar rates, $\$ / £$ and $\$ / €$.

behavior of the difference between the upper bound and the market implied (upper range) and the difference between the lower bound and the market implied (lower range). Their descriptive statistics across deltas are illustrated in Table 2. Both the level and variation of the upper ranges are larger than those of the lower ranges across deltas. We also investigate the relationships between the ranges and the correlation of two dollar rates as Proposition 2 suggests that the higher the correlation is, the closer the market implied volatility will be to the lower bound. We regress the upper range and the lower range, respectively, on the correlation and report the slope coefficient estimates in Table $2 .^{8}$ The results clearly indicate that the

\footnotetext{
${ }^{8}$ The correlation coefficients are estimated using the dynamic conditional correlation (DCC) multivariate GARCH model proposed by Engle (2002) with the historical time series data of two dollar spot rates. The fact that correlations between financial assets are usually time-varying has important implications in many ways such as portfolio hedging and multivariate asset pricing. This model overcomes the complexity of conventional multivariate GARCH models in computation by directly modeling the time-varying correlation as a conditional process. The procedure of using the DCC GARCH model to generate the time-varying correlation series is detailed in Engle (2002).
} 
Table 2

Summary statistics of the estimated upper ranges and lower ranges

\begin{tabular}{|c|c|c|c|c|c|c|c|}
\hline Delta & 0.9 & 0.75 & 0.63 & 0.5 & 0.37 & 0.25 & 0.1 \\
\hline \multicolumn{8}{|c|}{ Panel 1: Upper ranges } \\
\hline Mean & 0.1033 & 0.1065 & 0.1076 & 0.1086 & 0.1085 & 0.1082 & 0.1069 \\
\hline Standard deviation & 0.0208 & 0.0208 & 0.0206 & 0.0207 & 0.0205 & 0.0206 & 0.0206 \\
\hline$\beta$ & $(8.02)$ & $(8.35)$ & $(8.37)$ & $(8.24)$ & $(8.18)$ & $(7.96)$ & $(7.44)$ \\
\hline \multicolumn{8}{|c|}{ Panel 2: Lower ranges } \\
\hline \multirow[t]{2}{*}{$\beta$} & -0.0855 & -0.1121 & -0.1049 & -0.0958 & -0.0905 & -0.0869 & -0.0818 \\
\hline & $(-7.98)$ & $(-8.93)$ & $(-8.59)$ & $(-8.65)$ & $(-8.62)$ & $(-8.50)$ & $(-8.24)$ \\
\hline
\end{tabular}

This table consists of the summary statistics of the estimated upper ranges and lower ranges of the cross-rate (€/£) options across deltas. The upper ranges and lower ranges are the distances between the upper bounds and market implieds and between the lower bounds and market implieds, respectively. In addition, the parameter estimates of the following regression model are provided.

$R_{t}=\alpha+\beta \operatorname{Corr}_{t}+\varepsilon_{t}$,

where $R_{t}$ is the upper or lower range, Corr ${ }_{t}$ denotes the correlation between the two dollar rates, and $\varepsilon_{t}$ is the residual term at time $t$. The correlations are generated by the DCC model of Engle (2002). The numbers in the parentheses are $t$-statistics.

upper (lower) range is significantly positively (negatively) associated with the correlation of two dollar rates across deltas; i.e. the higher the correlation is, the closer the market implied will be to the lower bound. This finding is consistent with the analytical properties of our pricing bounds.

In summary, the lower bounds exhibit a smile shape while the upper bounds and the market implied volatilities are relatively flat across deltas. Both the upper and lower bounds exhibit tractable and persistent relationships with the market prices of cross-rate options.

\subsection{Pricing bounds, correlation, and the cross-rate option prices}

Since it has been found both analytically and empirically that there are persistent relationships between the market prices of the cross-rate options and our pricing bounds, we further use a regression model to measure the extent where the cross-rate option prices can be explained by our pricing bounds. We regress the market implied volatilities on the upper and lower bounds. ${ }^{9}$ The regression model is specified as

\footnotetext{
${ }^{9}$ The information content of our pricing bounds for cross-rate options is similar to that of the prices of options on the corresponding two dollar rates. Therefore, the implied volatilities of the two dollar rates have the potential to provide similar explanatory power for the cross-rate implied volatility as our bounds do. However, according to our analysis, the implied volatilities of $\$ / £$ and $\$ / €$ are highly correlated (about 0.8 ). Thus, a serious multicollinearity problem occurs when directly regressing the implied volatility of $€ / £$ on those of $\$ / £$ and $\$ / €$ although its adjusted $R^{2}$ is just slightly lower than Model 1. As our pricing bounds for cross-rate options are linear combinations of the prices of two dollar-rate options with particular strike prices, our bounds provide a solution to the multicollinearity problem by transforming two highly correlated implied volatilities to two less associated bounds. As a result, using the bounds instead of the dollar-rate implied volatilities enables our analysis to be more valid and reliable.
}

Model 1: $\quad \operatorname{MIV}_{t}=c+\beta_{1} \mathrm{UB}_{t}+\beta_{2} \mathrm{LB}_{t}+\varepsilon_{t}$

where $\mathrm{MIV}_{t}, \mathrm{UB}_{t}$, and $\mathrm{LB}_{t}$, respectively, denote the market implied volatility of the one-month cross-rate option on $€ / £$, the upper bound, and the lower bound at day $t$, and $\varepsilon_{t}$ is the residual term. ${ }^{10}$ The estimates for this model are shown in Panel 1 of Table 3.

From Panel 1 of Table 3, we find highly significant regression coefficients of $\beta_{1}$ and $\beta_{2}$. The adjusted $R^{2} \mathrm{~s}$ are very high and range from 0.72 to 0.77 across deltas. It is noticeable that $\beta_{1}$ adheres to a small range (between 0.33 and 0.39 ) while $\beta_{2}$ ranges from 0.26 to 0.59 . In other words, the upper bound contains almost the same level of information content for the cross-rate options across delta, while the lower bound contains different levels of information content across deltas. In short, we confirm that there are strong and stable relationships between the market prices of cross-rate options and the pricing bounds estimated from the market prices of the options on two dollar rates.

From the analytical properties in Proposition 2, we find that no correlation information is used in the calculation of the pricing bounds of the cross-rate options, for which we only utilize the price information of the options on two dollar rates individually. However, Driessen et al. (2006) analyze the relationship between the prices of stock index options and the prices of individual stock options included in the index, and they find the relevance of correlation risk and the associated premium for stock index options pricing. Inspired by their results, this paper includes an extra explanatory variable, the historical correlation of two dollar rates, into Model 1 to see whether the correlation is able to provide additional explanatory power. Thus, the regression model is modified as

\footnotetext{
${ }^{10}$ As the bounds are estimated every day from the one-month options, the data used for the regression model are daily data.
} 
Table 3

Explanatory power of estimated bounds and correlation to market implied volatility

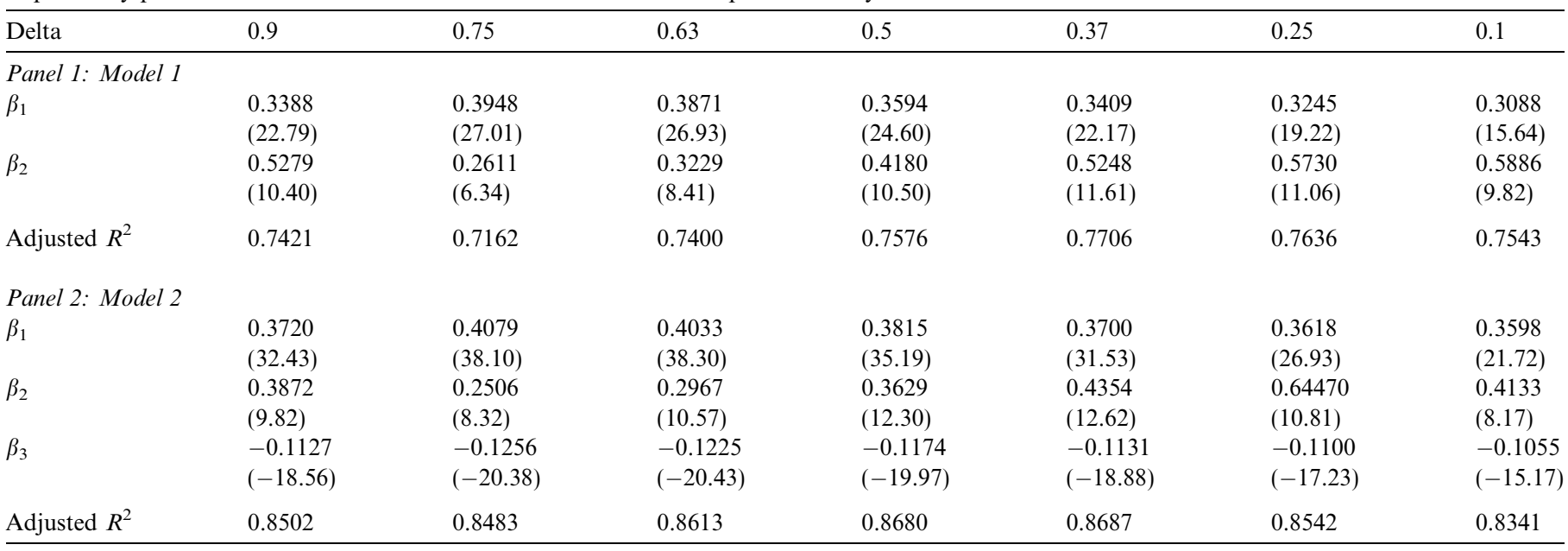

This table consists of the regression results of the following two models:

Model 1: $\mathrm{MIV}_{t}=c+\beta_{1} \mathrm{UB}_{t}+\beta_{2} \mathrm{LB}_{t}+\varepsilon_{t}$.

Model 2: $\mathrm{MIV}_{t}=c+\beta_{1} \mathrm{UB}_{t}+\beta_{2} \mathrm{LB}_{t}+\beta_{3} \operatorname{Corr}_{t}+\varepsilon_{t}$.

Here, $\mathrm{MIV}_{t}, \mathrm{UB}_{t}, \mathrm{LB}_{t}$, and $\mathrm{Corr}_{t}$ denote, respectively, the market implied volatility of an option on $€ / £$, the upper bound, the lower bound, and the historical DCC correlation between $\mathrm{S} / €$ and $\$ / £$ at day $t$, and $\varepsilon_{t}$ is the residual term. The numbers in the parentheses are $t$-statistics.

Model 2: $\quad \operatorname{MIV}_{t}=c+\beta_{1} \mathrm{UB}_{t}+\beta_{2} \mathrm{LB}_{t}+\beta_{3} \operatorname{Corr}_{t}+\varepsilon_{t}$,

where Corr $_{t}$ is the DCC correlation coefficients of two dollar rates at day $t$. When the correlation of two dollar rates increases, the variance of the cross-rate decreases and thus the cross-rate option price also decreases. Therefore, the regression coefficient of the historical correlation $\left(\beta_{3}\right)$ is expected to be negative.

The regression results for Model 2 are shown in Panel 2 of Table 3. It is clearly seen that the correlations of two dollar rates provide incremental information in explaining cross-rate option prices as all adjusted $R^{2} \mathrm{~s}$ increase by about $10 \%$ in comparison to Model 1 . The regression coefficients for the correlation across deltas are significantly negative and consistent with our expectation. Furthermore, our results are in line with the analyses and findings of Driessen et al. (2006).

As volatility is usually highly persistent, it is expected that including the one-period lagged volatility as an independent variable will increase the goodness-of-fit. However, in this study what we intend to investigate is how well the cross-rate option price can be explained by the dollar-rate price information only (i.e. without previous crossrate option price information), rather than how well the model can be specified. Therefore, we only use the estimated upper and lower bounds as the explanatory variables in this study. For comparison, we also include the one-period lagged cross-rate implied volatility as an additional explanatory variable in Model 2. The unreported results show that even with the additional explanatory variable which is highly correlated with the dependent variable, the coefficients of the upper and lower bounds and the correlation are still significant at the $1 \%$ level and their signs are still consistent with the theoretical expectations. ${ }^{11}$

Due to the significant in-sample explanatory power of the estimated bounds and correlation to the market prices of cross-rate options, we are interested in the performance of our empirical models in the out-of-sample prediction. Given the estimated parameters of the previous model (Model 2), we predict the current implied volatility for the cross-rate $(€ / £)$ options from the current market prices of the dollar-rate options and the historical correlation. The actual and predicted implied volatilities of the crossrate options for the delta of 0.5 are shown in Fig. 2. The results for other moneyness are very similar and thus omitted.

The prediction errors, defined as the absolute values of the actual values minus the predicted values of implied volatilities, from Model 2 are generally small. The average errors across deltas are about $0.3 \%$, which is smaller than the bid-ask spread in the OTC market. In addition, the volatilities of the prediction errors are very small as well (about $0.3 \%$ ), implying that the model performs consistently well across time.

In summary, the pricing bounds estimated from option prices of two dollar rates and the correlation of two dollar rates can provide highly significant information for explaining the cross-rate option prices across deltas. Our results are valuable since our pricing bounds (which are portfolios of dollar rate options) are applicable to practical usage not only for price explanation, but also for hedging,

\footnotetext{
11 As expected, the result has an almost perfect goodness-of-fit $\left(R^{2} \simeq 0.96\right.$ ) owing to correcting the considerable first-order serial correlation in the residuals of Model 2.
} 
Delta: 0.5

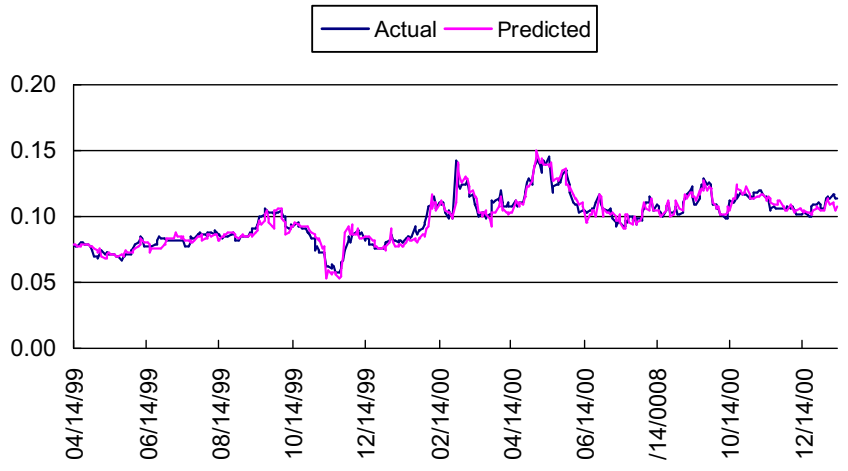

Fig. 2. Actual and predicted implied volatilities for cross-rate options. This figure consists of the evolution of the actual and predicted BlackScholes implied volatilities of the cross-rate $(€ / £)$ options with the delta of 0.5 . The actual implied volatilities are backed out from the market prices of options. The predicted implied volatilities are obtained from Model 2 in Section 4 using the market prices of options on two dollar rates, $\$ / £$ and $\$ /$ $€$, and the historical DCC correlation of two dollar rates.

particularly when the real-time cross-rate option prices are unobservable.

\subsection{Robustness analysis}

To investigate whether our results are robust, we first check whether the estimated bounds rely on the assumption of the RND for the dollar rates, and then we analyze whether the out-of-sample prediction errors from Model 2 are sensitive to sample selection, the implied volatility level, and changes in the curvature of the implied volatility function.

To check whether the estimated bounds depend on the distribution assumption of the dollar rate, we assume that the RNDs of two dollar rates follow the lognormal mixtures distribution and then compare the bounds estimated under this assumption with those under the GB2 distribution assumption. As shown in Table 4, the differences between the bounds estimated using these two different RND assumptions are statistically insignificant across deltas at the $10 \%$ significance level although the differences of the lower bounds are larger than those of the upper bounds.

To check whether sample selection affects our findings, we re-do the out-of-sample prediction of Model 2 for two evenly divided sub-samples. Although the prediction errors are slightly higher in the second sub-periods $(0.31 \%$ vs. $0.35 \%$ on average), the patterns across deltas are basically the same. In other words, our finding does not depend on the sample selection.

As the volatility of exchange rates increases over our sample period, it is natural to check whether the increasing volatility affects the accuracy of information provided by our pricing bounds. Moreover, although the average implied volatilities of all exchange rates exhibit a smile shape, the slopes of the implied volatility curves vary from being negatively sloped to positively sloped during our sample period. This implies that risk-neutral skewness and kurtosis change substantially every day. Therefore, to further check whether the out-of-sample prediction error of Model 2 depends on the volatility level or the change in the curvature of the implied volatility function, we first calculate the implied volatility, skewness, and kurtosis using Theorem 1 of Bakshi et al. (2003) and then run the following regression model:

$E_{t}=c+\alpha E_{t-1}+\beta X_{t}$,

where $E_{t}$ denotes the percentage prediction error and $X_{t}$ represents the implied volatility, skewness, or kurtosis level estimated using the approach of Bakshi et al. (2003) at time $t$. The AR(1) specification is motivated by the high first-order autocorrelation of prediction errors. The estimates are reported in Table 5. All $\beta$ coefficients are insignificant under the $10 \%$ significance level.

Fig. 3 indicates that the RNDs of the cross-rates are fattailed (average kurtosis equals 3.31 ) and slightly negatively skewed (average skewness equals -0.13 ). Fig. 3 also shows that the implied skewness changes noticeably over time. Nevertheless, Panel 2 of Table 5 suggests that there is no clear evidence supporting that the prediction errors across deltas are affected even though implied skewness changes much. Similarly, Panel 3 of Table 5 shows that the implied

Table 4

Robustness analysis for option bounds

\begin{tabular}{|c|c|c|c|c|c|c|c|}
\hline Delta & 0.9 & 0.75 & 0.63 & 0.5 & 0.37 & 0.25 & 0.1 \\
\hline \multicolumn{8}{|c|}{ Panel 1: Upper bounds } \\
\hline GB2 & 0.2087 & 0.2061 & 0.2056 & 0.2058 & 0.2064 & 0.2077 & 0.2118 \\
\hline Mixtures & 0.2086 & 0.2060 & 0.2056 & 0.2058 & 0.2065 & 0.2078 & 0.2119 \\
\hline \multicolumn{8}{|c|}{ Panel 2: Lower bounds } \\
\hline GB2 & 0.0405 & 0.0319 & 0.0302 & 0.0315 & 0.0352 & 0.0396 & 0.0478 \\
\hline
\end{tabular}

This table consists of the means of the upper and lower bounds of the cross-rate ( $€ / £)$ options across deltas, which are estimated using two different RND assumptions, GB2 and lognormal mixtures, for the dollar rates. In addition, the $p$-values of the mean equality tests for the two assumed distributions are provided. 
Table 5

Robustness analysis for out-of-sample prediction errors

\begin{tabular}{|c|c|c|c|c|c|c|c|}
\hline Delta & 0.9 & 0.75 & 0.63 & 0.5 & 0.37 & 0.25 & 0.1 \\
\hline \multicolumn{8}{|c|}{ Panel 1: Regression of errors on implied volatility } \\
\hline$\beta$ & $\begin{array}{l}0.0133 \\
(0.10)\end{array}$ & $\begin{array}{l}-0.0651 \\
(-0.55)\end{array}$ & $\begin{array}{l}-0.1138 \\
(-1.00)\end{array}$ & $\begin{array}{l}-0.1174 \\
(-1.02)\end{array}$ & $\begin{array}{l}-0.0495 \\
(-0.46)\end{array}$ & $\begin{array}{l}-0.0128 \\
(-0.12)\end{array}$ & $\begin{array}{l}0.0990 \\
(0.91)\end{array}$ \\
\hline \multicolumn{8}{|c|}{ Panel 2: Regression of errors on implied skewness } \\
\hline$\beta$ & $\begin{array}{l}0.0099 \\
(0.40)\end{array}$ & $\begin{array}{l}0.0027 \\
(0.12)\end{array}$ & $\begin{array}{l}0.0104 \\
(0.48)\end{array}$ & $\begin{array}{l}0.0119 \\
(0.54)\end{array}$ & $\begin{array}{l}0.0242 \\
(1.18)\end{array}$ & $\begin{array}{l}0.0399 \\
(1.96)\end{array}$ & $\begin{array}{l}0.0612 \\
(3.07)\end{array}$ \\
\hline
\end{tabular}

This table consists of the parameter estimates of the following regression model used to analyze whether the out-of-sample prediction errors for Model 2 depend on volatility, skewness or kurtosis.

$E_{t}=c+\alpha E_{t-1}+\beta X_{t}+\varepsilon_{t}$,

where $E_{t}$ denotes the prediction error in percentage and $X_{t}$ is the implied volatility, implied skewness, or implied kurtosis at time $t$ estimated using Theorem 1 of Bakshi et al. (2003). The numbers in the parentheses are $t$-statistics.

Skewness

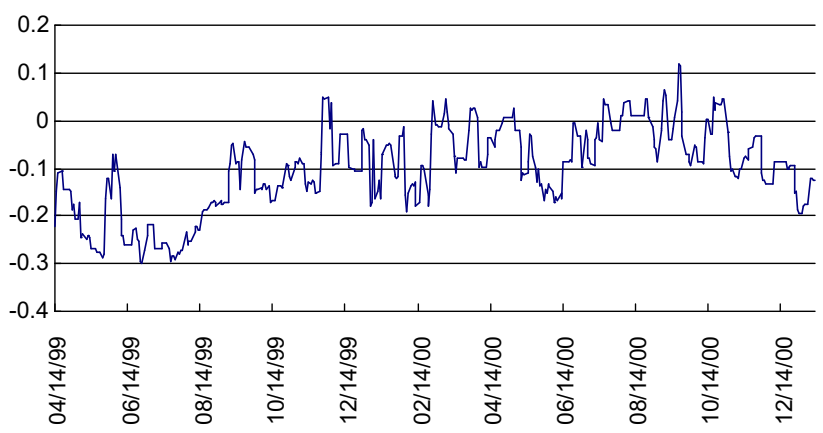

Kurtosis

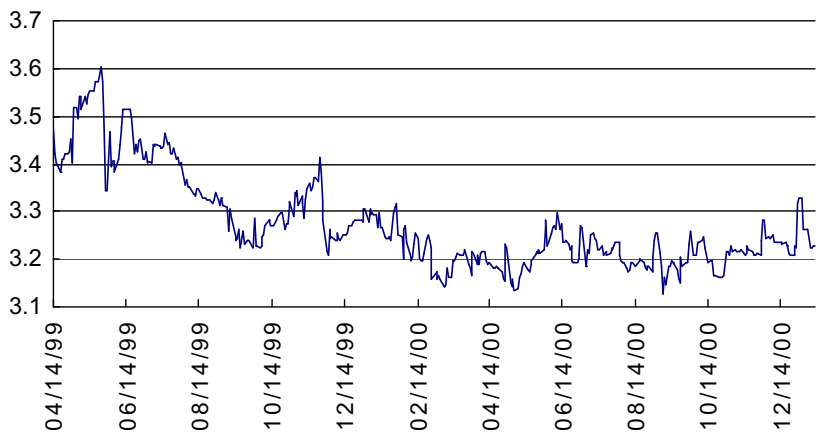

Fig. 3. Implied skewness and kurtosis for cross-rate options. This figure consists of the evolutions of the implied skewness and kurtosis of the cross-rate $(€ / £)$ options. The implied skewness and kurtosis are calculated using Theorem 1 of Bakshi et al. (2003). The results indicate that the riskneutral distributions of the cross-rates are fat-tailed (average kurtosis equals 3.31) and slightly negatively skewed (average skewness equals $-0.13)$.

kurtosis has little impact on the prediction errors across deltas.

In summary, the accuracy of information provided by our pricing bounds is immune to the market volatility level and the change in the curvature of the implied volatility function. Other proxies of the market volatility level, such as the implied volatilities for different moneyness levels, are also used and the results (not reported here) are almost unchanged.

\subsection{Bounds on delta of cross-rate options}

Given the estimated pricing bounds in terms of implied volatility, it is plausible to bound the cross-rate option's delta using our pricing bounds with Propositions 5 (or Proposition 6) of Bergman et al. (1996) when the call price of the cross-rate is unknown (or known).

We take the at-the-money (ATM) cross-rate call option traded on June 29, 1999 as an example and depict its delta bounds in Fig. 4. The solid lines in the descending order are the Black-Scholes prices computed as a function of the underlying asset price using volatilities of the upper bound, the market implied volatility, and the lower bound, respectively. When the cross-rate call price is unknown, its delta is bounded between 0.0261 and 0.9704 (dashed lines).

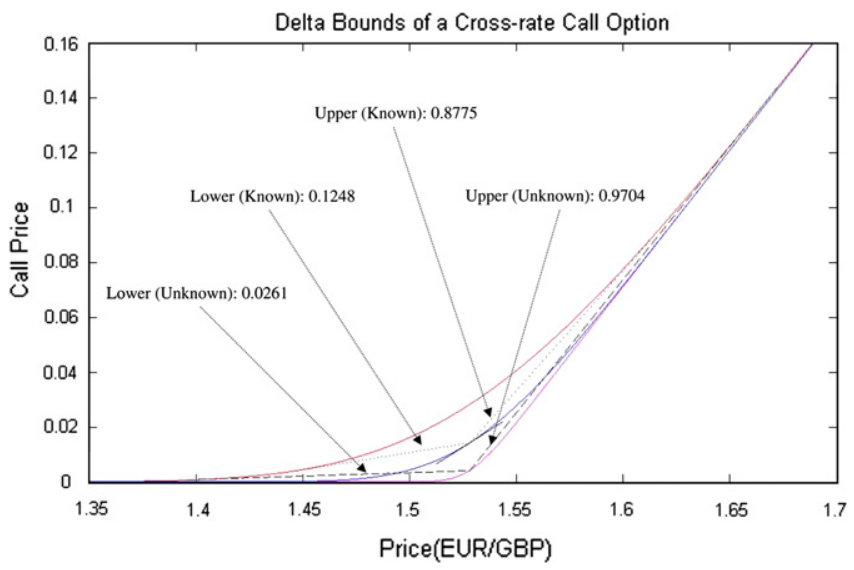

Fig. 4. Delta bounds of a cross-rate option. This figure consists of the upper and lower bounds on the delta of the ATM cross-rate $(€ / £)$ call option traded on June 29, 1999 when the cross-rate option price is unknown (dashed lines) or known (dotted lines). The delta bounds are calculated using the Propositions 5 and 6 of Bergman et al. (1996). 
When the call price is known, the delta bounds become tighter and ranges from 0.1248 to 0.8775 (dotted lines). ${ }^{12}$

\section{Concluding remarks}

Instead of pricing cross-rate options directly, this study relates the option pricing bounds to the prices of the corresponding dollar-rate options. Our pricing bounds are derived from a general result of the copula theory and thus do not rely on the distribution assumptions of state variables. Different from most option pricing bounds in the literature, our cross-rate option bounds are functions of the option prices (and sometimes also the spot prices) of two dollar rates.

Using the prices of options on foreign exchange rates among US dollar, euro, and pound sterling for the empirical tests, we show the persistent relationships between the market prices of the cross-rate $(€ / £)$ options and our pricing bounds. Our pricing bounds and the correlation between two dollar rates provide $85 \%$ of the information in explaining the prices of the cross-rate options. Therefore, our results are useful for risk management and derivative pricing, particularly for those having cross-rate risk exposures and when only the current option prices of two dollar rates are available.

The technique utilized to derive our cross-rate option pricing bounds can be applied to any European derivative security whose payoff can be rearranged as the same type as that of an exchange option. For example, one can derive the pricing bounds for quanto options using the copula approach applied in this paper. ${ }^{13}$ Further analyses related to the pricing bounds of the other type of exchange options are left to interested readers for future research.

\section{Acknowledgements}

We are indebted to anonymous referees, Joao Amaro de Matos, Antonio Camara and Bing-Huei Lin for helpful comments and suggestions; and also to the seminar participants at National Chengchi University, National Chiao Tung University, National Taiwan University, National Tsing Hua University, Yuan Ze University, the European Financial Management Association Annual Meeting 2006, the 4th Bachelier Finance Society World Congress, the Finance Management Association Annual Meeting 2006, the Taiwan Finance Association Annual Meeting 2006, and the National Taiwan University International Conference on Finance 2006. We thank the National Science Council of Taiwan for financial support and acknowledge the research assistance of Der-Fong Chen.

\footnotetext{
12 The results for other moneyness levels (defined as the strike prices divided by the forward price) are similar. For example, the delta of the call option with the moneyness level of 1.0325 (OTM) ranges from 0.0003 to 0.5986 (from 0.0127 to 0.5719 ) when the option price is unknown (known).

${ }^{13}$ The formulae of quanto option pricing bounds are also derived by the authors and are available upon request.
}

\section{Appendix A}

\section{Derivation of the price bounds for the cross-rate option}

Let $\operatorname{Pr}, F_{i}(x)$, and $r$ be the probability, the cumulative distribution function, and the dollar risk-free interest rate, respectively. Due to Breeden and Litzenberger (1978), the price of an option on the minimum of two risky assets can be expressed as

$$
\begin{aligned}
& \text { Call }_{\min }\left(S^{\$ / \epsilon}, K S^{\$ / \epsilon}, 0, t, T\right) \\
& =\mathrm{e}^{-r(T-t)} \int_{0}^{\infty} \operatorname{Pr}\left(\min \left(S^{\$ / \epsilon}, K S^{\$ / \epsilon}\right)>x\right) \mathrm{d} x \\
& =\mathrm{e}^{-r(T-t)} \int_{0}^{\infty} \operatorname{Pr}\left(S^{\$ / \epsilon}>x, K S^{\$ / \epsilon}>x\right) \mathrm{d} x \\
& =\mathrm{e}^{-r(T-t)} \int_{0}^{\infty} \bar{C}\left(\bar{F}_{S^{S / \varepsilon}}(x), \bar{F}_{K S^{S / \epsilon}}(x)\right) \mathrm{d} x,
\end{aligned}
$$

where $\bar{C}$ is a survival copula ${ }^{14}$ and $\bar{F}_{i}(x)=1-F_{i}(x)$. According to the Fréchet bounds in the copula theory, it is true that $\max (u+v-1,0) \leqslant \bar{C}(u, v) \leqslant \min (u, v)$ since $\bar{C}(u, v)$ is a copula. Consequently, the upper and lower bounds of the minimum option are given as the following, respectively:

$$
\begin{aligned}
& \text { Call }_{\text {min }}^{+}\left(S^{\$ / \epsilon}, K S^{S / \epsilon}, 0, t, T\right) \\
& \quad=\mathrm{e}^{-r(T-t)} \int_{0}^{\infty} \min \left(\bar{F}_{S^{S / \epsilon}}(x), \bar{F}_{K S^{S / \epsilon}}(x)\right) \mathrm{d} x, \\
& \text { Call }_{\text {min }}^{-}\left(S^{\$ / \epsilon}, K S^{S / \epsilon}, 0, t, T\right) \\
& \quad=\mathrm{e}^{-r(T-t)} \int_{0}^{\infty} \max \left(\bar{F}_{S^{S / \epsilon}}(x)+\bar{F}_{K S^{S / \epsilon}}(x)-1,0\right) \mathrm{d} x .
\end{aligned}
$$

Since $\bar{F}_{i}(u)$ is a decreasing function of $u$ and $K^{* *}$ is a constant which solves $\bar{F}_{S^{S / \ell}}\left(K^{* *}\right)+\bar{F}_{K S^{s / \epsilon}}\left(K^{* *}\right)=1$, it is true that $\bar{F}_{S^{s / \mathfrak{E}}}(u)+\bar{F}_{K S^{S / \ell}}(u) \geqslant 1$ for $u \leqslant K^{* *}$. Therefore, the lower bound of the minimum option is

$$
\begin{aligned}
\text { Call }_{\text {min }}^{-} & \left(S^{\S / \epsilon}, K S^{S / \epsilon}, 0, t, T\right) \\
= & \mathrm{e}^{-r(T-t)} \int_{0}^{\infty} \max \left(\bar{F}_{S^{S / \epsilon}}(u)+\bar{F}_{K S^{S / \epsilon}}(u)-1,0\right) \mathrm{d} u \\
= & \mathrm{e}^{-r(T-t)} \int_{0}^{K^{* *}} \bar{F}_{S^{S / \epsilon}}(u) \mathrm{d} u+\mathrm{e}^{-r(T-t)} \\
& \times \int_{0}^{K^{* *}} \bar{F}_{K S^{S / \epsilon}}(u) \mathrm{d} u-\mathrm{e}^{-r(T-t)} \int_{0}^{K^{* *}} \mathrm{~d} u \\
= & \mathrm{e}^{-r(T-t)} \int_{0}^{\infty} \bar{F}_{S^{S / \epsilon}}(u) \mathrm{d} u-\mathrm{e}^{-r(T-t)} \int_{K^{* *}}^{\infty} \bar{F}_{S^{S / \epsilon}}(u) \mathrm{d} u \\
& +\mathrm{e}^{-r(T-t)} \int_{0}^{\infty} \bar{F}_{K S^{S / \epsilon}}(u) \mathrm{d} u-\mathrm{e}^{-r(T-t)} \\
& \times \int_{K^{* *}}^{\infty} \bar{F}_{K S^{S / \epsilon}}(u) \mathrm{d} u-\mathrm{e}^{-r(T-t)} \int_{0}^{K^{* *}} \mathrm{~d} u
\end{aligned}
$$

\footnotetext{
${ }^{14}$ If two uniform variables $U$ and $V$ are jointed with a copula function $C$, then the joint probability that $U$ and $V$ are greater than $u$ and $v$, respectively, is given by a survival function:
}

$\operatorname{Pr}(U>u, V>v)=1-u-v+C(u, v)=\bar{C}(1-u, 1-v)$. 
$$
=S_{t}^{\$ / £} \mathrm{e}^{-r_{\mathfrak{£}}(T-t)}-\operatorname{Call}\left(S^{\$ / £}, K^{* *}, t, T\right)+K S_{t}^{\$ / \epsilon} \mathrm{e}^{-r_{\epsilon}(T-t)}
$$$$
-\operatorname{Call}\left(K S^{\$ / \epsilon}, K^{* *}, t, T\right)-\mathrm{e}^{-r(T-t)} K^{* *} \text {. }
$$

Substituting Eq. (A.3) into Eq. (2) and applying the putcall parity, we obtain the upper bound of the cross-rate option price as

$$
\begin{aligned}
\operatorname{Call}_{\$}^{€ /\left.\right|^{+}} & =S_{t}^{\$ / €} \mathrm{e}^{-r_{\mathfrak{£}}(T-t)}-\mathrm{Call}_{\text {min }}^{-}\left(S^{\$ / £}, K S^{\$ / \epsilon}, 0, t, T\right) \\
& =\operatorname{Call}\left(S^{\$ / £}, K^{* *}, t, T\right)+\operatorname{Put}\left(K S^{\$ / \epsilon}, K^{* *}, t, T\right) \\
& =\operatorname{Call}\left(S^{\$ / £}, K^{* *}, t, T\right)+K \operatorname{Put}\left(S^{\$ / \epsilon}, K^{\prime \prime}, t, T\right),
\end{aligned}
$$

where $K^{\prime \prime}=K^{* *} / K$.

Assume that there exists a constant $K^{*}$ such that $\bar{F}_{S^{s / \mathrm{E}}}\left(K^{*}\right)=\bar{F}_{K S^{s / \epsilon}}\left(K^{*}\right)$. If $\bar{F}_{S^{s / \mathrm{E}}}(u)<\bar{F}_{K S^{s / \epsilon}}(u)$ for $u<K^{*}$, then it is straightforward to show that the upper bound of the minimum option is

$$
\begin{aligned}
& \mathrm{Call}_{\min }^{+}\left(S^{\$ / \epsilon}, K S^{\$ / \epsilon}, 0, t, T\right) \\
& =\mathrm{e}^{-r(T-t)} \int_{0}^{\infty} \min \left(\bar{F}_{S^{S / \mathrm{E}}}(u), \bar{F}_{K S^{\mathrm{s} / \mathrm{e}}}(u)\right) \mathrm{d} u \\
& =\mathrm{e}^{-r(T-t)} \int_{0}^{K^{*}} \bar{F}_{S^{\mathrm{s} / \mathrm{\epsilon}}}(u) \mathrm{d} u+\mathrm{e}^{-r(T-t)} \int_{K^{*}}^{\infty} \bar{F}_{K S^{\mathrm{s} / \epsilon}}(u) \mathrm{d} u \\
& =\mathrm{e}^{-r(T-t)} \int_{0}^{\infty} \bar{F}_{S^{S / \mathfrak{E}}}(u) \mathrm{d} u-\mathrm{e}^{-r(T-t)} \int_{K^{*}}^{\infty} \bar{F}_{S^{S / \mathrm{f}}}(u) \mathrm{d} u \\
& +\mathrm{e}^{-r(T-t)} K \int_{K^{*} / K}^{\infty} \bar{F}_{S^{S / \epsilon}}(u) \mathrm{d} u \\
& =S_{t}^{\$ / £} \mathrm{e}^{-r_{\mathrm{\epsilon}}(T-t)}-\operatorname{Call}\left(S^{\$ / £}, K^{*}, t, T\right)+K \operatorname{Call}\left(S^{\$ / €}, K^{\prime}, t, T\right),
\end{aligned}
$$

where $K^{\prime}=K^{*} / K$. Substituting Eq. (A.5) into Eq. (2) yields the lower price bound of the cross-rate option as

$$
\begin{aligned}
\operatorname{Call}_{\$}^{€ / \mathfrak{E}^{-}} & =S_{t}^{\$ / £} \mathrm{e}^{-r_{\mathfrak{£}}(T-t)}-\mathrm{Call}_{\min }^{+}\left(S^{\$ / £}, K S^{\$ / \epsilon}, 0, t, T\right) \\
& =\operatorname{Call}\left(S^{\$ / £}, K^{*}, t, T\right)-K \operatorname{Call}\left(S^{\$ / \epsilon}, K^{\prime}, t, T\right) .
\end{aligned}
$$

Similarly, if $\bar{F}_{S^{s / \varepsilon}}(u)>\bar{F}_{K S^{s / \epsilon}}(u)$ for $u<K^{*}$, then one can derive that

$$
\begin{aligned}
& \operatorname{Call}_{\text {min }}^{+}\left(S^{\$ / £}, K S^{\$ / \epsilon}, 0, t, T\right)=K S_{t}^{\$ / \epsilon} \mathrm{e}^{-r_{\epsilon}(T-t)} \\
& \quad-K \operatorname{Call}\left(S^{\$ / \epsilon}, K^{\prime}, t, T\right)+\operatorname{Call}\left(S^{\$ / €}, K^{*}, t, T\right), \\
& \operatorname{Call}_{\$}^{€ / \mathcal{E}^{-}}=S_{t}^{\$ / €} \mathrm{e}^{-r_{\mathfrak{\epsilon}}(T-t)}-K S_{t}^{\$ / \epsilon} \mathrm{e}^{-r_{\epsilon}(T-t)} \\
& \quad+K \operatorname{Call}\left(S^{\$ / \epsilon}, K^{\prime}, t, T\right)-\operatorname{Call}\left(S^{\$ / €}, K^{*}, t, T\right) .
\end{aligned}
$$

\section{Appendix B}

\section{Proof of Proposition 2}

When the two dollar rates follow a bivariate lognormal distribution, $\bar{F}_{S^{\mathrm{s} / \mathrm{E}}}(K)$ is actually the risk-neutral probability that the European call option on $\$ / £$ with a strike price of $K$ will be exercised, because

$$
\begin{aligned}
\bar{F}_{S^{\zeta / \mathfrak{t}}}(K) & =1-F_{S^{S / \mathfrak{f}}}(K)=1-\operatorname{Pr}\left(S_{T}^{S / \mathfrak{f}}<K\right) \\
& =N\left(d_{2}\left(S_{t}^{\S / \mathfrak{£}}, K, r_{\S}, r_{\mathfrak{f}}, \sigma_{\$ / \mathfrak{f}}, \tau\right)\right),
\end{aligned}
$$

where $\tau=T-t$ and $d_{2}(\cdot)=\frac{\ln \left(S_{t}^{S / \mathscr{t}} / K\right)+\left(r_{\mathrm{S}}-r_{\mathrm{E}}-\frac{1}{2} \sigma_{\mathrm{S} / \mathrm{t}}^{2}\right) \tau}{\sigma_{\mathrm{S} / \mathrm{f}} \sqrt{\tau}}$. Therefore, $K^{* *}$ in Proposition 1 is a constant satisfying that

$$
\begin{aligned}
& N\left(d_{2}\left(S_{t}^{\$ / £}, K^{* *}, r_{\$}, r_{£}, \sigma_{\$ / \mathfrak{f}}, \tau\right)\right) \\
& +N\left(d_{2}\left(K S_{t}^{\$ / \epsilon}, K^{* *}, r_{\$}, r_{\epsilon}, \sigma_{\$ / \epsilon}, \tau\right)\right)=1 .
\end{aligned}
$$

Since $N(x)+N(-x)=1$ is true, one can show that

$$
d_{2}\left(S_{t}^{\$ / €}, K^{* *}, r_{\$}, r_{\mathfrak{£}}, \sigma_{\$ / \mathfrak{\epsilon}}, \tau\right)=-d_{2}\left(K S_{t}^{\$ / €}, K^{* *}, r_{\$}, r_{€}, \sigma_{\$ / €}, \tau\right) .
$$

Thus, the solution of for $K^{* *}$ is

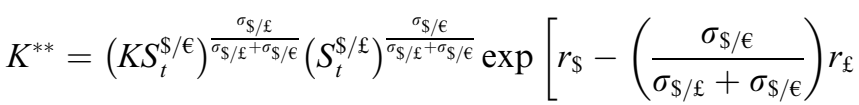

$$
\begin{aligned}
& \left.-\left(\frac{\sigma_{\$ / £}}{\sigma_{\$ / €}+\sigma_{\$ / \epsilon}}\right) r_{\epsilon}-\frac{\sigma_{\$ / \AA} \sigma_{\$ / \epsilon} \tau}{2}\right] .
\end{aligned}
$$

From Eqs. (3) and (A.8), the upper bound is given by

$$
\begin{aligned}
& S_{t}^{\$ / £} \mathrm{e}^{-r_{\mathfrak{f}} \tau} N\left(d_{1}\left(S_{t}^{\$ / £}, K^{* *}, r_{\$}, r_{£}, \sigma_{\$ / \mathfrak{f}}, \tau\right)\right) \\
& -K^{* *} \mathrm{e}^{-r_{\S} \tau} N\left(d_{2}\left(S_{t}^{\$ / \mathfrak{f}}, K^{* *}, r_{\$}, r_{\mathfrak{f}}, \sigma_{\$ / \mathfrak{f}}, \tau\right)\right) \\
& +K^{* *} \mathrm{e}^{-r_{S} \tau} N\left(-d_{2}\left(K S_{t}^{\$ / \epsilon}, K^{* *}, r_{\$}, r_{\epsilon}, \sigma_{\$ / \epsilon}, \tau\right)\right) \\
& -K S_{t}^{\$ / \epsilon} \mathrm{e}^{-r_{\epsilon} \tau} N\left(-d_{1}\left(K S_{t}^{\$ / \epsilon}, K^{* *}, r_{\$}, r_{\epsilon}, \sigma_{\$ / \epsilon}, \tau\right)\right) \\
& =S_{t}^{\$ / £} \mathrm{e}^{-r_{\mathfrak{£}} \tau} N\left(d_{1}\left(S_{t}^{\$ / \mathfrak{£}}, K^{* *}, r_{\$}, r_{\mathfrak{£}}, \sigma_{\$ / \mathfrak{t}}, \tau\right)\right) \\
& -K S_{t}^{\$ / \epsilon} \mathrm{e}^{-r_{\epsilon} \tau} N\left(-d_{1}\left(K S_{t}^{\$ / \epsilon}, K^{* *}, r_{\$}, r_{\epsilon}, \sigma_{\$ / \epsilon}, \tau\right)\right) \text {. }
\end{aligned}
$$

Note that the above upper bound is denominated in US dollars and its value in euros is

$$
\begin{aligned}
& S_{t}^{€ / £} \mathrm{e}^{-r_{\mathfrak{E}} \tau} N\left(d_{1}\left(S_{t}^{\$ / £}, K^{* *}, r_{\$}, r_{\mathfrak{f}}, \sigma_{\$ / \mathfrak{f}}, \tau\right)\right) \\
& -K \mathrm{e}^{-r_{\epsilon} \tau} N\left(-d_{1}\left(K S_{t}^{\$ / €}, K^{* *}, r_{\$}, r_{€}, \sigma_{\$ / €}, \tau\right)\right) .
\end{aligned}
$$

Substituting Eq. (A.9) into Eq. (A.10) yields the upper bound, i.e.

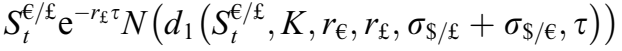

$$
\begin{aligned}
& -K \mathrm{e}^{-r_{\epsilon} \tau} N\left(d_{2}\left(S_{t}^{€ / £}, K, r_{€}, r_{£}, \sigma_{\$ / €}+\sigma_{\$ / €}, \tau\right)\right) \\
& =C_{\mathrm{BS}}\left(S_{t}^{€ / £}, K, r_{€}, r_{\mathfrak{£}}, \sigma_{\$ / £}+\sigma_{\$ / €}, \tau\right) \text {. }
\end{aligned}
$$

For brevity, we derive the lower bound only for the case where $\sigma_{\$ / \mathfrak{\varepsilon}}>\sigma_{\$ / \epsilon}$. Since $K^{*}$ satisfies that $N\left(d_{2}\left(S_{t}^{\$ / \mathfrak{t}}\right.\right.$, $\left.\left.K^{*}, r_{\S}, r_{£}, \sigma_{\$ / \epsilon}, \tau\right)\right)=N\left(d_{2}\left(K S_{t}^{\$ / \epsilon}, K^{*}, r_{\$}, r_{\epsilon}, \sigma_{\$ / \epsilon}, \tau\right)\right)$, one can show that its solution is

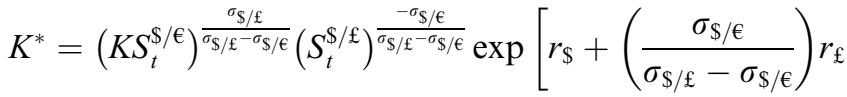

$$
\begin{aligned}
& \left.-\left(\frac{\sigma_{\$ / £}}{\sigma_{\$ / £}-\sigma_{\$ / \epsilon}}\right) r_{€}+\frac{\sigma_{\$ / £} \sigma_{\$ / \epsilon} \tau}{2}\right] .
\end{aligned}
$$

Since $\sigma_{\$ / €}>\sigma_{\$ / \epsilon}$, it is straightforward to show that when $u<K^{*}$, 


$$
\begin{aligned}
N & \left(d_{2}\left(S_{t}^{\$ / £}, u, r_{\$}, r_{\mathfrak{£}}, \sigma_{\$ / \mathfrak{f}}, \tau\right)\right) \\
& =N\left(d_{2}\left(S_{t}^{\$ / \epsilon}, K^{*}, r_{\$}, r_{\mathfrak{£}}, \sigma_{\$ / \mathfrak{f}}, \tau\right)+\frac{\ln \left(K^{*} / u\right)}{\sigma_{\$ / \mathfrak{f}} \sqrt{\tau}}\right) \\
& <N\left(d_{2}\left(K S_{t}^{\$ / \epsilon}, K^{*}, r_{\$}, r_{\epsilon}, \sigma_{\$ / \epsilon}, \tau\right)+\frac{\ln \left(K^{*} / u\right)}{\sigma_{\$ / €} \sqrt{\tau}}\right) \\
& =N\left(d_{2}\left(K S_{t}^{\$ / \epsilon}, u, r_{\$}, r_{\epsilon}, \sigma_{\$ / \epsilon}, \tau\right)\right) .
\end{aligned}
$$

Therefore, the lower bound is determined by the first alternative of Eq. (4), ${ }^{15}$ i.e.

$$
\begin{aligned}
& S_{t}^{\$ / £} \mathrm{e}^{-r_{\mathfrak{f}} \tau} N\left(d_{1}\left(S_{t}^{\$ / £}, K^{*}, r_{\$}, r_{£}, \sigma_{\$ / \mathfrak{f}}, \tau\right)\right) \\
& -K^{*} \mathrm{e}^{-r_{\S} \tau} N\left(d_{2}\left(S_{t}^{\$ / £}, K^{*}, r_{\$}, r_{£}, \sigma_{\$ / \mathfrak{t}}, \tau\right)\right) \\
& -K S_{t}^{\$ / \epsilon} \mathrm{e}^{-r_{\epsilon} \tau} N\left(d_{1}\left(K S_{t}^{\$ / \epsilon}, K^{*}, r_{\$}, r_{\epsilon}, \sigma_{\$ / \epsilon}, \tau\right)\right) \\
& +K^{*} \mathrm{e}^{-r_{\$} \tau} N\left(d_{2}\left(K S_{t}^{\$ / \epsilon}, K^{*}, r_{\$}, r_{€}, \sigma_{\$ / \epsilon}, \tau\right)\right) \\
& =S_{t}^{\$ / £} \mathrm{e}^{-r_{\mathfrak{f}} \tau} N\left(d_{1}\left(S_{t}^{\$ / \mathfrak{f}}, K^{*}, r_{\$}, r_{\mathfrak{£}}, \sigma_{\$ / \mathfrak{f}}, \tau\right)\right) \\
& -K S_{t}^{\$ / \epsilon} \mathrm{e}^{-r_{\epsilon} \tau} N\left(d_{1}\left(K S_{t}^{\$ / \epsilon}, K^{*}, r_{\$}, r_{\epsilon}, \sigma_{\$ / \epsilon}, \tau\right)\right) .
\end{aligned}
$$

The above lower bound is denominated in US dollars and its value in euros is

$$
\begin{aligned}
& S_{t}^{€ / £} \mathrm{e}^{-r_{£} \tau} N\left(d_{1}\left(S_{t}^{\$ / £}, K^{*}, r_{\$}, r_{\mathfrak{£}}, \sigma_{\$ / £}, \tau\right)\right) \\
& \quad-K \mathrm{e}^{-r_{\ell} \tau} N\left(d_{1}\left(K S_{t}^{\$ / €}, K^{*}, r_{\$}, r_{€}, \sigma_{\$ / €}, \tau\right)\right) .
\end{aligned}
$$

Substituting Eq. (A.11) into Eq. (A.12) yields the lower bound, i.e.

$$
\begin{aligned}
S_{t}^{€ / £} \mathrm{e}^{-r_{£} \tau} N\left(d_{1}\left(S_{t}^{€ / £}, K, r_{€}, r_{£}, \sigma_{\$ / £}-\sigma_{\$ / €}, \tau\right)\right) \\
\quad-K \mathrm{e}^{-r_{€} \tau} N\left(d_{2}\left(S_{t}^{€ / £}, K, r_{€}, r_{£}, \sigma_{\$ / £}-\sigma_{\$ / €}, \tau\right)\right) \\
\quad=C_{\mathrm{BS}}\left(S_{t}^{€ / £}, K, r_{€}, r_{£}, \sigma_{\$ / £}-\sigma_{\$ / €}, \tau\right) .
\end{aligned}
$$

\section{References}

Bakshi, G., Madan, D., 2000. Spanning and derivative-security valuation. Journal Financial Economics 55, 205-238.

Bakshi, G., Kapadia, N., Madan, D., 2003. Stock return characteristics, skew laws, and the differential pricing of individual equity options. Review of Financial Studies 16, 101-143.

Bakshi, G., Carr, P., Wu, L., forthcoming. Stochastic risk premiums, stochastic skewness in currency options, and stochastic discount factors in international economies. Journal of Financial Economics.

Basso, A., Pianco, P., 1997. Decreasing absolute risk aversion and option pricing bounds. Management Science 43, 206-216.
Bergman, T., Grundy, B., Weiner, Z., 1996. General properties of option prices. Journal of Finance 51, 1573-1610.

Bliss, R.R., Panigirtzoglou, N., 2002. Testing the stability of implied probability density functions. Journal of Banking and Finance 26, 381-422.

Bookstaber, R.M., McDonald, J.B., 1987. A general distribution for describing security price returns. Journal of Business 60, 401-424.

Breeden, D., Litzenberger, R., 1978. Prices of state-contingent claims implicit in options prices. Journal of Business 51, 621-651.

Carr, P., Ellis, K., Gupta, V., 1998. Static hedging of exotic options. Journal of Finance 53, 1165-1190.

Cherubini, U., Luciano, E., Vecchiato, W., 2004. Copula Methods in Finance. John Wiley and Sons.

Driessen, J., Maenhout, P., Vilkov, G., 2006. Option-implied correlations and the price of correlation risk. Working paper, University of Amsterdam and INSEAD

Engle, R.F., 2002. Dynamic conditional correlation: A simple class of multivariate generalized autoregressive conditional heteroskedasticity models. Journal of Business and Economic Statistics 20, 339-350.

Garman, M., 1976. An algebra for evaluating hedging portfolios. Journal of Financial Economics 3, 403-428.

Grundy, B.D., 1991. Option prices and the underlying asset's return distribution. Journal of Finance 46, 1045-1070.

Jackwerth, J.C., Rubinstein, M., 1996. Recovering probability distributions from option prices. Journal of Finance 51, 1611-1631.

Joe, H., 1997. Multivariate Models and Dependence Concepts. Chapman \& Hall.

Levy, H., 1985. Upper and lower bounds of call and put option value: Stochastic dominance approach. Journal of Finance 40, 1197 1217.

Mathur, K., Ritchken, P., 2000. Minimum option prices under decreasing absolute risk aversion. Review of Derivative Research 3, 135-156.

Melick, W., Thomas, C., 1997. Recovering an asset's implied PDF from option prices: An application to crude oil during the Gulf crisis Journal of Financial and Quantitative Analysis 32, 91-115.

Merton, R.C., 1973. Theory of rational option pricing. Bell Journal of Economics and Management Science 4, 141-183.

Nelsen, R.B., 1999. An Introduction to Copulas. Springer, New York.

Ritchey, R., 1990. Call option valuation for discrete normal mixtures. Journal of Financial Research 13, 285-295.

Ritchken, P., 1985. On option pricing bounds. Journal of Finance 40, 1219-1233.

Ritchken, P., Kuo, S., 1989. On stochastic dominance and decreasing absolute risk averse option pricing bounds. Management Science 35, 51-59.

Ryan, P., 2003. Progressive option bounds from the sequence of currently expiring options. European Journal of Operation Research 151, 193 223.

Taylor, S.J., Wang, Y.-H., 2005. Option prices and risk neutral densities for currency cross-rates. Working paper, Lancaster University and National Central University.

${ }^{15}$ On the other hand, if the volatility of $\$ / £$ is smaller than that of $\$ / €$, then the lower bound is determined by the second alternative of Eq. (4). 